395 MENOPAUSAL SYMPTOMS AND SEXUAL DISORDERS IN EPITHELIAL OVARIAN CANCER SURVIVORS, A GINECO VIVROVAIRE2 STUDY

${ }^{1}$ Florence Joly, ${ }^{2}$ Anne Gompel, ${ }^{3}$ Raffaèle Fauvet, ${ }^{4}$ Idlir Licaj, ${ }^{5}$ Djhhane Ahmed-Lecheheb, ${ }^{4}$ Jean-Michel Grellard, ${ }^{6}$ Elsa Kalbacher, ${ }^{7}$ Olivier Tredan, ${ }^{1}$ Francois Gernier, ${ }^{8}$ Patricia Pautier. ${ }^{1}$ Centre François Baclesse; Unité Inserm 1086; ${ }^{2}$ Chu Paris Centre - Hôpital Cochin, Site Cochin; ${ }^{3}$ Chu Caen Departement OF Gynecology AND Obstetrics; ${ }^{4}$ Centre Francois Baclesse; ${ }^{5}$ Unite Inserm 1086 Anticipe; ${ }^{6}$ Chu Jean Minjoz; ${ }^{7}$ Centre Leon Berard; ${ }^{8}$ Gustave Roussy

10.1136/ijgc-2020-ESGO.167

Introduction/Background We have previously shown that Epithelial Ovarian Cancer (EOC) and its treatments have significant negative effects on Quality of Life (QoL) and long term fatigue. The aim of the present multicentric VIVROVAIRE2 study was to report the main menopausal symptoms of Epithelial Ovarian Cancer survivors (EOCS).

Methodology One hundred sixty-six patients of the 322 EOCS without relapse $\geq 3$ years after first line of treatment accepted to participate to a gynecological consultation carried out by a gynecologist, including a questionnaire related to menopausal symptoms (MS), sexuality, clinical examination, and osteodensitometry. MS (hot flashes and/or night sweats) were described according to natural menopause (NM) or surgically induced menopause (SIM). QoL, fatigue, insomnia and mood disorders were measured with the questionnaires (FACT-G, FACIT Fatigue, ISI, and HADS).

Results Median age was 62 years [20-83], FIGO stage III/IV $(48 \%)$ and $<10 \%$ BRCA1\&2 mutated. Histological subtypes were: high grade serous $28 \%$, low grade serous $22 \%$, endometrioid G2-3 (15\%) endometriod G1 ( $3 \%$ ), clear-cell 21\%, mucinous 5\%. All EOCS had surgery, 97\% of patients received platinum and taxane chemotherapy, median delay from treatment was 5 years [3-24] and 59 (36\%) had SIM. $14 \%$ of EOCS had osteoporosis. Half of patients reported MS either hot flashes (47\%) or night sweats (32\%). 72\% with SIM had MS compared to $41 \%$ with NM $(\mathrm{p}<.001)$. MS were not associated with poor global QoL, fatigue, insomnia or mood disorders. At the gynecological consultation, two-thirds of EOCS reported a decrease in sexual desire notably EOCS with SIM, wich showed a greater decreased libido than NM $(\mathrm{p}<.02)$.

One hundred seven patients have never been treated with Hormone Replacement Therapy (HRT) including 59 who reported MS and 48 who (40\%) had SIM.

Among 85 EOCS with MS, 80 (94\%) (38 SIM and 42 NM) did not benefit from HRT after cancer treatment; $76 \%$ presented no CI of HRT.

Conclusion Menopausal symptoms and sexual disorders are frequently reported by EOCS, particularly among surgically induced menopause patients. A majority of EOCS with MS may beneficiate from HRT to improve these symptoms.

Disclosures The authors declare that they have no conflict of interest in relation to the subject of the article.

\section{EFFECTIVENESS OF PHOTODYNAMIC THERAPY IN LICHEN SCLEROSUS WITH NEOPLASTIC BACKGROUND}

${ }^{1}$ Magdalena Bizoń-Szpernalowska, ${ }^{2}$ Danuta Maślińska, ${ }^{3}$ Włodzimierz Sawicki. ${ }^{1}$ Chair and Department of Obstetrics, Gynecology and Gyncological Oncology; ${ }^{2}$ Medical University in Warsaw; ${ }^{3}$ Chair and Department of Obstetrics, Gynecology and Gynecological Oncology, Medical University of Warsaw

10.1136/ijgc-2020-ESG0.168
Introduction/Background The main problem connected with lichen sclerosus is inflammatory proccess and correlation of this vulvar dermatose with autoimmunological and neoplastic diseases. Photodynamic therapy is one of the most popular worldwide discussed method of treatment of lichen sclerosus. Despite the short history, this method has a quiet good benefits.

The aim of the study was to assess the influence of neoplastic disease on effectiveness of photodynamic therapy used in lichen sclerosus treatment.

Methodology Analysis was based on 182 patients with age 31-88 from Outpatient Vulvar Clinic, which is a part of Department of Obstetrics, Gynecology and Gynecological Oncology. Material was divided into 3 groups: patients with neoplastic disease in history, women with positive familiar history of neoplastic disease and women neither medical history nor familiar history of neoplastic diseases.

Analysis was based on immunohistochemical reaction in samples of vulvar biopsy with receptors TLR, mast cells, Langerhans cells and MeCP2 - Methyl-CpG-binding protein 2, vulvoscopic assessment with special scales our own authorship before and 10 weeks after photodynamic therapy and questionnaire of patients' vulvar symptoms before and after the treatment.

Results TLR3, TLR9 and Langerhans cells take part in advanced stage of lichen sclerosus pathogenesis. MeСР2 occurs in every stage of lichen sclerosus. Localization depends on different stage of disease and can be in every layer of the skin. The role of this protein is to take part in epigenetics process.

In vulvoscopic assessment, the percentage of improvement after PDT was higher in group of women with no correlation to neoplastic diseases $(55,3 \%)$ than in women with contact with neoplastic disease $(47,7 \%)$.

According to subjective symptoms connected with lichen sclerosus the most frequent was itching, which stand for $76,2 \% ; 71,7 \%$ and $66,9 \%$ in following groups. In comparison to other complains, the highest percentage of every group were patient with neoplastic disease in the past. Satisfaction with the level above $50 \%$ after photodynamic therapy reffered $80,8 \%$ patients from all analysed population.

Conclusion To sum up, the presence of neoplastic disease can influence on tissue and clinical phenotype on effectiveness of photodynamic therapy used in treatment of lichen sclerosus. Disclosures

\section{HOW TO RECONSTRUCT AN OPEN ABDOMINAL WALL AFTER NECROTIZING FASCIITIS: SURGICAL MANAGEMENT IN DIFFICULT CASES}

${ }^{1}$ Seda Sahin Aker, ${ }^{2}$ Arda Özdemir, ${ }^{1}$ Frrat Ortaç. ${ }^{1}$ Ankara University Faculty of Medicine; Gynecologic Oncology; ${ }^{2}$ Ankara University Faculty of Medicine; Plastic and Reconstructive Surgery

\subsection{6/ijgc-2020-ESGO.169}

Introduction/Background Necrotizing fasciitis (NF) is a rare but very fatal infection involving and causing necrosis of the subcutaneous tissue and fascia. The incidence of NF is $0.4 /$ 100000. NF has a high mortality rate so it is needed an early diagnosis and proper treatment.There are several risk factors of NF. NF presents as painful, patchy discoloration of the skin without margins and a black necrotic plaque at the wound area. Ischemia and tissue necrosis can develop and local anaesthesia can occur because of the nerve damage.

Methodology A 59-year-old Turkish woman was admitted to hospital with a complaint of a postmenouposal bleeding. 